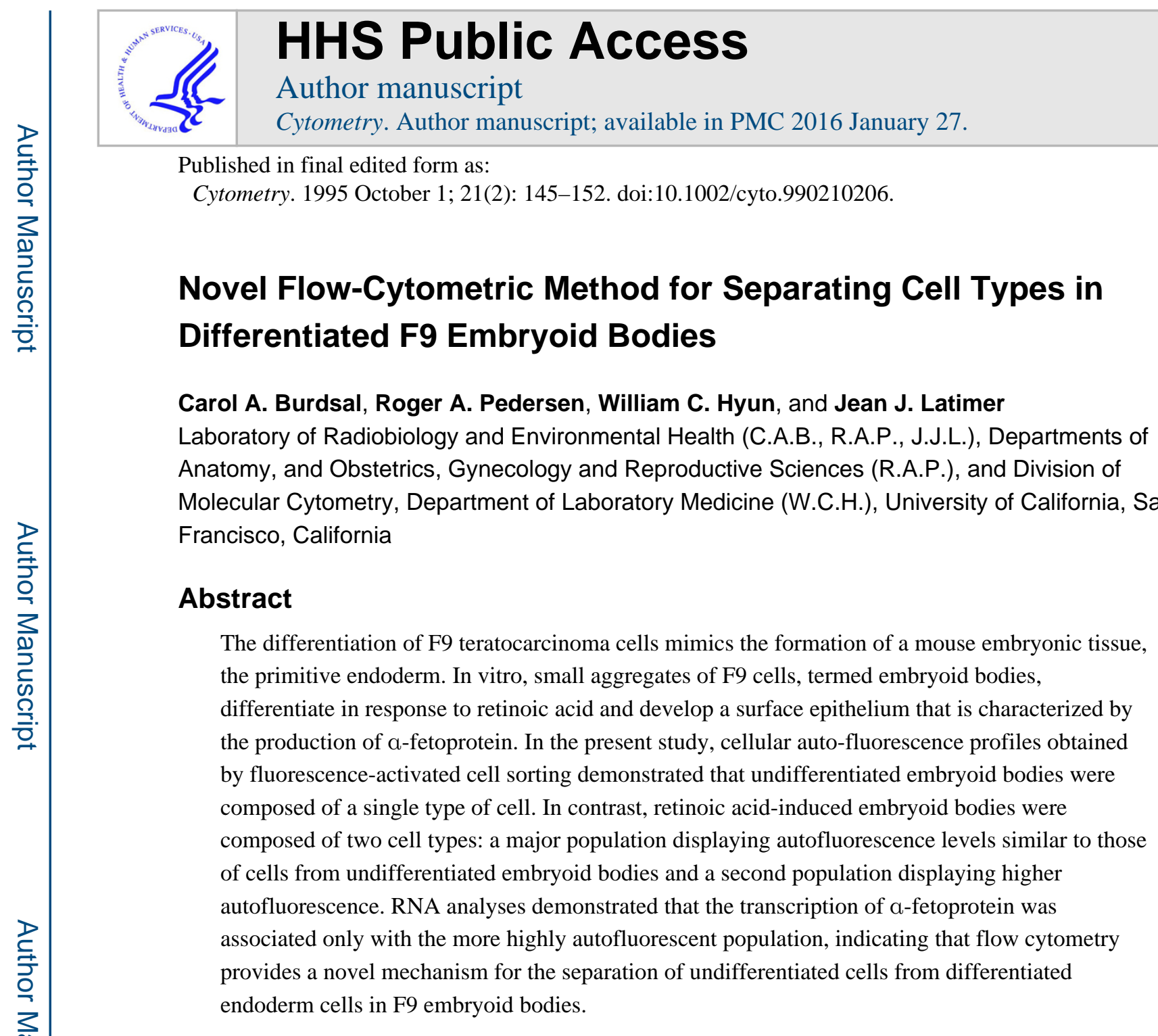

\title{
Key terms
}

a-Fetoprotein; autofluorescence; differentiation; embryonal carcinoma cells; fluorescenceactivated cell sorting; retinoic acid; visceral endoderm

\begin{abstract}
At the blastocyst stage of development in the mouse, an epithelium, the primitive endoderm, differentiates over the surface of the inner cell mass, which alone will give rise to definitive embryonic tissues (21). Tissues derived from the primitive endoderm, the visceral and parietal yolk sacs, will provide vital support for the developing mouse embryo. Because of the small size of the blastocyst, which is $100 \mu \mathrm{m}$ in diameter and composed of approximately 128 cells, it is difficult to isolate substantial quantities of primitive endoderm. The same difficulties are encountered in attempts to isolate derivatives of the primitive endoderm, visceral endoderm or parietal endoderm, from late blastocyst stage embryos. The need for substantial quantities of tissues for molecular and cell biological analysis has
\end{abstract}

\footnotetext{
Address reprint requests to Dr. Carol A. Burdsal, Department of Cell and Molecular Biology, 2000 Percival Stern Hall, Tulane University, New Orleans, LA 70118.

Dr. Latimer's present address is Department of Obstetrics, Gynecology and Reproductive Sciences, Magee-Womens Research Institute, Room 430, University of Pittsburgh, Pittsburgh, PA 15213.
} 
prompted investigators to turn to model systems of early mammalian development. The best characterized system for the in vitro differentiation of the early extraembryonic tissues of the mouse is teratocarcinoma cells.

In suspension culture, aggregates of cells from the F9 teratocarcinoma line form spherical structures known as embryoid bodies (16). Addition of the morphogen retinoic acid (RA) to such cultures induces a differentiation event, the formation of an outer epithelium on the surface of the embryoid bodies (22). This outer epithelial layer expresses urokinase-type plasminogen activator and cytokeratins Endo A and Endo B (1, 11, 15). Embryonically, these markers are expressed by the primary endoderm and the visceral endoderm; accordingly, it is generally accepted that the outer epithelium of differentiated embryoid bodies is endodermal in nature. The secreted glycoprotein a-fetoprotein (AFP), which functions as a carrier of hormones and charged molecules, is also expressed by the visceral endoderm in the embryo $(9,12)$. In RA-induced embryoid bodies, both AFP protein and transcripts have been localized uniquely to the outer endodermal cells $(3,11,19)$. Isolation of cell types from embryoid bodies would provide the means to identify novel cell-specific products whose expression could be assayed in either the inner cell mass or endoderm of the blastocyst. This approach would provide valuable in-sights into the mechanism of early differentiation in the mouse embryo.

Commonly used separation techniques, such as cell panning and cell isolation by means of magnetic beads, rely on a cell-type-specific antibody for selecting the cells to be collected. Other methods rely on intrinsic characteristics of the cells, such as separation by density in Percoll gradients. This technique has been used to isolate endoderm cells from differentiated embryoid bodies (6). In this report we describe differences in autofluorescence between cells in RA-induced embryoid bodies and demonstrate that cell types in differentiated embryoid bodies can be separated by flow cytometry according to these differences. Differences in cellular autofluorescence have been used previously to discriminate between various cell types, including alveolar macrophages from other accessory cells in the human lung and blood monocytes from alveolar macrophages $(8,17)$. This sorting protocol offers the advantage over density-based separation that any number of parameters, such as cell size, cellular autofluorescence, and external and internal cellular morphology, can be used solely or in combination for the separation of cell types. In addition, the precision of electronic collection is greater than that generally achieved by the manual collections of cells from gradients. We also purified intact steady-state RNA from the isolated cell populations and confirmed the identity of the two populations by the expression of AFP, an endodermspecific marker, on RNA blots.

\section{MATERIALS AND METHODS}

\section{Cell Culture and Differentiation}

Monolayer cultures of the mouse F9 cell line (5) were maintained on 100-mm tissue culture dishes (Falcon Labware, Oxnard CA) coated with $0.1 \%$ gelatin (Sigma Chemical Co, St. Louis, MO) F9 cells were cultured in Dulbecco's modified Eagle's medium supplemented with $10 \%$ fetal calf serum (Hyclone, Logan, UT), $20 \mathrm{mM}$ glucose, $2 \mathrm{mM}$ glutamine (Gibco/ BRL, Gaithersburg, MD), $100 \mathrm{U} / \mathrm{ml}$ penicillin and $10 \mu \mathrm{g} / \mathrm{ml}$ streptomycin (Gibco/BRL), and 
$10^{-4} \mathrm{M} \beta$-mercaptoethanol (Sigma). Monolayer cultures were passaged every two to three days.

For the production of embryoid bodies, F9 monolayers were trypsinized ( $0.05 \%$ trypsin in the presence of $0.02 \%$ EDTA), and the cell suspension was seeded onto $100-\mathrm{mm}$ bacteriological petri dishes (Fisher Scientific, Santa Clara, CA) Cell aggregates formed and within one to two d developed into spherical aggregates that grew suspended in the F9 medium. These embryoid bodies remained undifferentiated until they were induced to form an endodermal epithelium by culturing for $8 \mathrm{~d}$ in $\mathrm{F} 9$ medium with the addition of $5 \times 10^{-8} \mathrm{M}$ RA (Sigma). Suspension cultures of embryoid bodies were fed every one to two d.

\section{Histology}

For histology, undifferentiated and RA-induced embryoid bodies were fixed for $1 \mathrm{~h}$ to overnight in 2\% glutaraldehyde (Sigma) in phosphate-buffered saline (PBS). Fixed embryoid bodies were dehydrated by sequential incubation in 50, 70, 90, and 100\% ethanol. Dehydrated embryoid bodies were embedded in Polaron Embedding medium (Energy Beam Sciences, Inc., Agawam, MA). Four-micrometer sections were stained with Gill's No. 5 hematoxylin and $0.6 \%$ eosin containing $0.1 \%$ phyloxine. Slides were viewed on an inverted Zeiss microscope and were photographed with Kodak Tri-X 400 ASA film.

\section{Antibodies and Immunocytochemistry}

A rabbit polyclonal serum against mouse AFP was obtained from ICN Immunobiologicals (Lisle, IL). A fluorescein-conjugated anti-rabbit secondary antibody was obtained from Sigma.

For immunocytochemistry, embryoid bodies were fixed in 99:1 ethanol:acetic acid (20), dehydrated through $100 \%$ ethanol and xylene(s), embedded in paraffin at $56^{\circ} \mathrm{C}$, and sectioned. Rehydrated slides were incubated in PBS with $10 \%$ milk proteins (Carnation nonfat dry milk, Carnation Co., Los Angeles, CA) to reduce non-specific, background fluorescence. Samples were incubated with the anti-AFP polyclonal antibody diluted 1:40 in PBS plus $10 \%$ fetal calf serum for $1 \mathrm{~h}$ at ambient temperature, washed three times in PBS plus 10\% fetal calf serum, then incubated for $45 \mathrm{~min}$ at ambient temperature in a 1:30 dilution of the fluorescein-conjugated anti-rabbit secondary antibody. Samples were washed three times in PBS plus 10\% fetal calf serum before viewing. Immunofluorescence was observed and photographed with a Zeiss microscope equipped with epifluorescence.

\section{Preparation of Cells for Flow Cytometry}

Cells were isolated from two types of embryoid bodies for cell sorting: unlabeled embryoid bodies and embryoid bodies labeled with fluorescein conjugated to isothiocyanate (FITC). Labeling of embryoid bodies involved washing them once with PBS and then resuspending the approximately $300-500 \mu \mathrm{l}$ of packed embryoid bodies in $10 \mathrm{ml}$ of PBS to which FITCconjugated wheat germ agglutinin (WGA-FITC, Sigma) was added to a final concentration of $10 \mu \mathrm{g} / \mathrm{ml}$. The labeled embryoid bodies were washed once with an excess of PBS before dissociation to remove unbound WGA-FITC. WGA-FITC-labeled embryoid bodies were dissociated to a single cell suspension by incubation for $5 \mathrm{~min}$ at $37^{\circ} \mathrm{C}$ in $0.05 \%$ trypsin and 
$0.02 \%$ EDTA and by gentle trituration. Trypsinization was stopped by the addition of medium containing $10 \%$ fetal calf serum. Cells were collected by centrifugation ( $5 \mathrm{~min}$, $250 \mathrm{~g}$ clinical centrifuge, International Equipment Co., Needham, MA) and were resuspended in PBS and filtered once through $43 \mu \mathrm{m}$ nylon mesh to remove clumped cells in preparation for flow analysis and sorting. Cell suspensions of unlabeled F9 undifferentiated embryoid bodies and unlabeled RA-induced embryoid bodies were prepared in the same way as controls for establishing sorting parameters.

\section{Flow Cytometry}

Cell preparations were analyzed and sorted by using a fluorescence-activated cell sorter (FACS) model 440 (Becton Dickinson, San Jose, CA) equipped with a $5 \mathrm{~W}$ argon ion laser (Spectra Physics, Mountain View, CA) elliptical focusing lens and a 70- $\mu \mathrm{m}$ nozzle orifice. The $488 \mathrm{~nm}$ line of the laser was used to excite the fluorescein with $400 \mathrm{~mW}$ of laser power. A $530 \mathrm{~nm} / 30 \mathrm{~nm}$ width band pass filter (Omega Optical, Brattleboro, VT) was used to filter the emitted fluorescence. Acquisition was triggered on forward scatter with a size threshold selected to minimize small debris. An unlabeled F9 sample was used to set an autofluorescence baseline and to establish forward and orthogonal scatter parameters. Three data parameters, forward and orthogonal scatter and fluorescence, were collected in the list mode and were analyzed by the software program Electric Desk (Stanford University, Palo Alto, CA) to establish appropriate sort windows. Sorting was done by using an 11 -drop delay with three deflected drops to maintain high purity. A FACScan (Becton Dickinson) was used to re-analyze the purity of sorted populations.

\section{RNA Analysis}

Upon collection of 100,000-500,000 cells into PBS, the sorted fractions of cells were centrifuged (10 min, $250 \mathrm{~g}$ ), and total RNA from the cell pellet was immediately extracted by the guanidine isothiocyanate-cesium gradient procedure (7) Total RNA from the fetal and adult livers of ICR mice (Charles River Laboratories, Wilmington, MA) was extracted in the same manner and used as a positive and negative control, respectively, on RNA blots RNA concentrations were determined by absorbance at $260 \mathrm{~nm}$. RNAs were fractionated on a 1.5\% agarose gel containing $2.2 \mathrm{M}$ formaldehyde and transferred to nitrocellulose. A 969 basepair insert isolated from a full length cDNA encoding mouse AFP (gift of Dr. S. Tilghman, Princeton University, Princeton, NJ) was labeled via the random priming reaction (18) and used to hybridize to RNA blots. RNA blots were washed at standard stringency ( 0.3 $\mathrm{M} \mathrm{NaCl}$ at $65^{\circ} \mathrm{C}$ ). Kodak XAR-5 film was used for autoradiography. RNA loading on gels was quantified by densitometric scanning of RNA blots hybridized with a probe specific for human triose phosphate isomerase (gift of Dr. H. Mohrenweiser, Lawrence Livermore National Laboratory, Livermore, CA). The expression of AFP was also quantified by scanning multiple exposures of RNA blots. 


\section{RESULTS}

\section{Morphological and Immunocytochemical Characterization of Embryoid Body Differentiation}

Histological analyses revealed that undifferentiated F9 embryoid bodies were composed of a uniform cell type (Fig. 1A,C). When induced to differentiate for $8 \mathrm{~d}$ in the presence of RA, the embryoid bodies produced an outer epithelial layer that was clearly demarcated from the core of the embryoid body (Fig. 1B,D). Cells in the core of RA-induced embryoid bodies morphologically resembled the cells in undifferentiated embryoid bodies (Fig. 1D). In contrast, the outer endoderm cells of RA-induced embryoid bodies displayed a novel morphology, that of a pseudostratified columnar epithelium. In addition, immunofluorescence assays demonstrated that only cells in this outer epithelium expressed the endoderm-specific, secreted glycoprotein AFP (Fig. 2).

\section{Flow-Cytometric Analysis of Unlabeled F9 Embryoid Bodies}

Differences in cellular morphology between core cells and endoderm cells were also apparent during flow-cytometric analyses. These cytometric analyses revealed that cells from undifferentiated F9 embryoid bodies displayed a uniform level of relatively low autofluorescence, and the distribution of these cells was described by a simple Gaussian curve (Fig. 3A). The autofluorescent profile of cells from RA-induced samples showed low autofluorescence, similar to that of undifferentiated cells, but an additional peak of higher autofluorescence was also present (Fig. 3B).

\section{Flow-Cytometric Analysis of WGA-FITC-Labeled F9 Embryoid Bodies}

In other experiments, embryoid bodies were labeled with WGA-FITC before dissociation. WGA-FITC binds N-acetyl glucosamine residues, and staining of embryoid bodies with this FITC-labeled lectin allows the fluorescent signal from cells to be better separated from that of debris present as a result of trypsinization. (The majority of debris is represented by the fluorescent signal adjacent to the Y-axis in Fig. 3.) Cytometric analyses demonstrated that WGA-FITC-labeled undifferentiated F9 embryoid bodies were composed of a single cell type that displayed a unimodal curve upon analysis. WGA-FITC-labeled RA-induced embryoid bodies were composed of two cell types, one with a relatively lower fluorescent signal and one with a relatively higher one. These profiles are similar to those of unlabeled embryoid bodies and indicate that, although the WGA-FITC label was added before dissociation, this regimen appears to have raised the level of fluorescence detected on all cell types (both inner and outer). The gate settings used for sorting unlabeled populations based on autofluorescent signals, and labeled populations based on total fluorescent signals, are indicated on the histograms.

These sorting profiles were highly reproducible. Details from the cytometric analysis of four separate experiments are presented in Table 1. Through multiple trials, the level of fluorescence (indicated by the peak position along the X-axis) of the low and high fluorescent populations in RA-induced embryoid bodies remained constant (Table 1A). In addition, the proportion of cells that were present in one peak compared with the other 
remained constant (Table 1B). Therefore, the fluorescent profiles were an innate characteristic of the differentiated cells that composed the embryoid bodies.

\section{Confirmation of Separation of Cell Populations}

To ensure that pure populations of cells were sorted based on our sort windows, we mixed collected samples of low and high fluorescent cells in equal numbers and reanalyzed the sample (Fig. 4). The second analysis clearly demonstrated that the newly mixed sample contained two populations of cells, centered on two fluorescent values separated by approximately 700 units on the X-axis with no overlap (Fig. 4B). As shown by the sorting gates in Fig. 4A, we chose conservative limits for collection of these cell populations (i.e, cells in the region where the two peaks might overlap were not collected). This re-analysis demonstrated the purity provided by our sorting parameters.

\section{RNA Analyses of Cell Populations Isolated by Flow Cytometry}

Upon collection of separated cell populations, total RNA was isolated and RNA analyses were performed. To determine the purity of the collected cells, we chose to assay the expression of the endoderm-specific transcript AFP. Because mouse fetal liver is known to express AFP (23), a dilution series of mouse fetal mRNA was included as a positive standard for densitometric scanning (Fig. 5, lanes 1-3), and adult liver, which does not express AFP, was included as a negative control (lane 11). Loading was normalized by densitometric scanning of the triose phosphate isomerase (TPI) signal; TPI is a general cellular metabolic enzyme whose level of transcription does not change with the differentiation of F9 teratocarcinoma cells. The results of the RNA analysis shown in Fig. 5 are representative of multiple experiments. For comparison with sorted populations, RNA isolated from intact undifferentiated and intact RA-induced embryoid bodies were included in the analyses.

Abundant AFP expression was manifested by intact RA-induced embryoid bodies (approximately $15 \mu \mathrm{g}$ RNA, Fig. 5, lane 5), whereas AFP expression from undifferentiated F9 embryoid bodies was undetectable by RNA analysis (15 $\mu \mathrm{g}$ RNA, lane 4). Based on densitometric quantification of known RNA standards (fetal and adult liver RNA), our RNA blots can detect between 50 and 100 copies of RNA per cell, per lane. Significantly, after separating WGA-FITC-labeled cells from RA-induced embryoid bodies based on their relative levels of fluorescence, AFP expression was detected only in the more highly fluorescent population (lane 9, approximately $\mu$ g RNA loaded).

The high autofluorescent population of unlabeled, RA- induced embryoid bodies (lane 7, approximately $1 \mu \mathrm{g}$ RNA loaded on gel) also showed abundant AFP expression; however, the low fluorescent population (lane 6) did not have detectable RNA present for comparison (see lower panel). RNA from low fluorescent cell populations in unlabeled embryoid bodies was routinely more difficult to obtain in high enough quantities for RNA analyses. This was probably due to the proximity of this cell population to cellular debris during flow sorting. During collection, fragments of cells or other debris may have registered as collection events, thus lowering the expected yield of RNA per cell. Therefore, WGA-FITC-labeling of cells, which moved both the low and high autofluorescent populations farther away from the 
debris area, was necessary for reproducible isolation of intact RNA from the low autofluorescent population. These results clearly demonstrate that cells expressing AFP can be completely separated from cells not expressing AFP in RA-induced embryoid bodies by flow cytometry.

\section{DISCUSSION}

We separated two populations of cells within RA-induced embryoid bodies by flow cytometry. RNA analyses demonstrated that intact steady-state mRNAs could be isolated from sorted populations from WGA-FITC-labeled embryoid bodies. AFP mRNA was detected in only one cell population, the higher autofluorescent population that concomitantly appears with the RA-induced differentiation of embryoid bodies. Numerous studies have demonstrated that AFP protein and mRNA are present only in the endoderm layer of differentiated embryoid bodies $(1,3,9,11,12,19)$. Therefore, we conclude that by successfully separating AFP-expressing cells from cells that do not express AFP, we have separated endoderm cells from core cells based on autofluorescent differences.

Autofluorescence probably reflects such properties as the cell's protein constitution and the presence of intracellular NADH, riboflavin, flavin coenzymes, flavoproteins, and metabolic rates within the cell $(2,4)$. The visceral endoderm is a specialized absorptive and secretive epithelium; correlating with these functions, outer endodermal cells of embryoid bodies contain numerous pinocytotic and lysosomal vacuoles $(10,12)$. This specialized ultrastructure, along with metabolic differences, may account for the differences in autofluorescence observed between endodermal cells and undifferentiated F9 cells.

To quantify the level of enrichment for cells that express AFP, we performed densitometric analyses (data not shown), in which the level of AFP expression was normalized with respect to the level of expression of a glycolytic gene, TPI. Scans indicated that the normalized level of AFP expression in highly autofluorescent populations was approximately 2.8 fold higher than that in intact RA-induced embryoid bodies. This enrichment approaches the theoretical limit of enrichment for this system, which can be calculated as follows. Highly autofluorescent (endoderm) cells constitute approximately $1 / 3$ of a differentiated embryoid body ( Table 1). In addition, we quantified the number of AFPpositive cells in the outer endoderm in our immunofluorescence assays and in published in situ hybridizations, and determined that approximately $1 / 3$ of outer endoderm cells express AFP mRNA or protein after $8 \mathrm{~d}$ of differentiation. Therefore, the fraction of cells expressing AFP in an intact differentiated embryoid body is $1 / 9$. After sorting, within samples of highly autofluorescent cells, $1 / 3$ of these would still be the AFP-expressing cells. Therefore, the theoretical limit for enrichment of AFP-producing cells after sorting based on autofluorescence would be (fraction of cells expressing AFP in sorted populations) $\div$ (fraction of cells expressing AFP in intact differentiated embryoid bodies), or $(1 / 3) \div(1 / 9)$, or 3 -fold. This value closely corresponds to our densitometric calculations. In addition, as the RNA blots demonstrated (Fig. 5), collections of the low auto-fluorescent cell population, which corresponds to the core, undifferentiated cells of the embryoid bodies, were not contaminated with cells that expressed AFP. Both the isolated endodermal cells and the undifferentiated cells would provide valuable material for biochemical and molecular 
analyses. Casanova et al. (6) isolated core cells and endoderm cells on Percoll gradients and demonstrated that endoderm cells expressed AFP after culture. However, they did not show that only one of the two separated populations expressed AFP at the time of isolation, as we have done.

In our analyses, lysates from separate collections were pooled, and the RNA was isolated by cesium gradient centrifugation, which greatly aids in preventing contamination of samples with DNA. In preparations in which $\geq 10^{6}$ cells were pooled, the yield of RNA was substantially higher than that of the preparations of 500,000 cells. This reduced yield in preparations of low cell number is probably due to the loss of RNA in the cesium gradients during centrifugation. This loss might best be minimized by the addition of carrier RNA when possible (i.e., if RNAs are to be analyzed on RNA blots rather than used for the generation of cDNA).

In conclusion, teratocarcinoma cells have proved to be a valuable resource for identifying gene products such as uvomorulin (E-cadherin), a protein necessary for compaction in the mouse embryo, and genes, such as the REX-1 gene, that respond to RA $(13,14)$. The technical difficulty in obtaining purified tissues from early mouse embryos remains a hindrance to advances in molecular investigations and provides continued impetus for the use of embryonal carcinoma cells as a resource. Novel techniques using these systems, such as the one described here, should facilitate the investigation of gene expression during early mammalian development.

\section{Acknowledgments}

This work was supported by the Program for Analytical Cytology at UCSF and the Lawrence Livermore Laboratories, an NIEHS Training Grant T32ES07106 (C.A.B., J.J.L.), NIH Grant Po-1 HD26732, and the Office of Health and Environmental Research, US Department of Energy, Contract DE-AC03-76-SF01012. and a research fellowship from the American Heart Association, California affiliate.

We gratefully acknowledge Mary McKenney and Dr. Stephen G. Grant (University of Pittsburgh, Pittsburgh, PA) for critical reading of the manuscript and for helpful discussions.

\section{LITERATURE CITED}

1. Adamson, EA.; Grover, A. The production and maintenance of a functioning epithelial layer from embryonal carcinoma cells. In: Silver, LM.; Martin, GR.; Strickland, S., editors. Teratocarcinoma Stem Cells. Cold Spring Harbor Press; Cold Spring Harbor, NY: 1983. p. 69-81.

2. Aubin JE. Autofluorescence of viable cultured mammalian cells. J Histochem Cytochem. 1979; 27:36-43. [PubMed: 220325]

3. Becker S, Casanova J, Grabel LB. Localization of endoderm-specific mRNAs in differentiating F9 embryoid bodies. Mech Devel. 1992; 37:3-12. [PubMed: 1376612]

4. Benson RC, Meyer RA, Zaruba ME, McKhann GM. Cellular autofluorescence-is it due to flavins? J Histochem Cytochem. 1979; 27:44-48. [PubMed: 438504]

5. Bernstine EG, Hooper ML, Grandchamp S, Ephrussi B. Alkaline phosphatase activity in mouse teratoma. Proc Natl Acad Sci USA. 1973; 70:3899-3903. [PubMed: 4521215]

6. Casanova JE, Grabel LB. The role of cell interactions in the differentiation of teratocarcinomaderived parietal and visceral endoderm. Dev Biol. 1988; 129:124-139. [PubMed: 2457525]

7. Chirgwin JM, Przybyla AE, MacDonald RJ, Rutter WJ. Isolation of biologically active ribonucleic acid from sources enriched in ribonuclease. Biochemistry. 1979; 18:5294-5299. [PubMed: 518835] 
8. Dethloff LA, Lehnert BE. Pulmonary interstitial macrophages: isolation and flow cytometric comparisons with alveolar macrophages and blood monocytes. J Leuk Biol. 1988; 43:80-90.

9. Dziadek M, Adamson ED. Localization and synthesis of alphafoeto-protein in post-implantation mouse embryos. J Embryol Exp Morphol. 1978; 43:289-313. [PubMed: 75937]

10. Freeman, SJ. Functions of extraembryonic membranes. In: Copp, AJ.; Cockroft, DL., editors. Postimplantation Mammalian Embryos. A Practical Approach. IRL Press; New York: 1990. p. 249-265.

11. Grover A, Oshima RG, Adamson ED. Epithelial layer formation in differentiating aggregates of F9 embryonal carcinoma cells. J Cell Biol. 1983; 96:1690-1696. [PubMed: 6343402]

12. Hogan BLM, Taylor A, Adamson E. Cell interactions modulate embryonal carcinoma cell differentiation into parietal or visceral endoderm. Nature. 1981; 291:235-237. [PubMed: 6164928]

13. Hosler BA, LaRosa GJ, Grippo JF, Gudas LJ. Expression of REX-1, a gene containing zinc finger motifs, is rapidly reduced by retinoic acid in F9 teratocarcinoma cells. Mol Cell Biol. 1989; 9:5623-5629. [PubMed: 2511439]

14. Hyafil F, Morello D, Babinet C, Jacob F. A cell surface glycoprotein involved in the compaction of embryonal carcinoma cells and cleavage stage embryos. Cell. 1980; 21:927-934. [PubMed: 6159985]

15. Marotti KR, Belin D, Strickland S. The production of distinct forms of plasminogen activator by mouse embryonic cells. Dev Biol. 1982; 90:154-159. [PubMed: 6174380]

16. Martin GR. Teratocarcinomas and mammalian embryogenesis. Science. 1980; 209:768-776. [PubMed: 6250214]

17. Nicod LP, Lipscomb MF, Toews GB, Weissler JC. Separation of potent and poorly functional human lung accessory cells based on autofluorescence. J Leuk Biol. 1989; 45:458-465.

18. Rigby PWJ, Dieckmann M, Rhodes C, Berg P. Labeling deoxyribonucleic acid to high specific activity in vitro by nick translation with DNA polymerase I. J Mol Biol. 1977; 113:237-251. [PubMed: 881736]

19. Rogers MB, Watkins SC, Gudas LJ. Gene expression in visceral endoderm: a comparison of mutant and wild-type F9 embryonal carcinoma cell differentiation. J Cell Biol. 1990; 110:17671777. [PubMed: 1692330]

20. Sainte-Marie G. A paraffin embedding technique for studies employing immunofluorescence. J Histochem Cytochem. 1962; 10:250-256.

21. Snell, GD.; Stevens, LC. Early embryology. In: Green, EL., editor. Biology of the Laboratory Mouse. 2. McGraw-Hill; New York: 1966. p. 205-245.

22. Strickland S, Mahdavi V. The induction of differentiation in teratocarcinoma stem cells by retinoic acid. Cell. 1978; 15:393-403. [PubMed: 214238]

23. Tilghman SM, Belayew A. Transcriptional control of the murine albumin/a-fetoprotein locus during development. Proc Natl Acad Sci USA. 1982; 79:5254-5257. [PubMed: 6182563] 

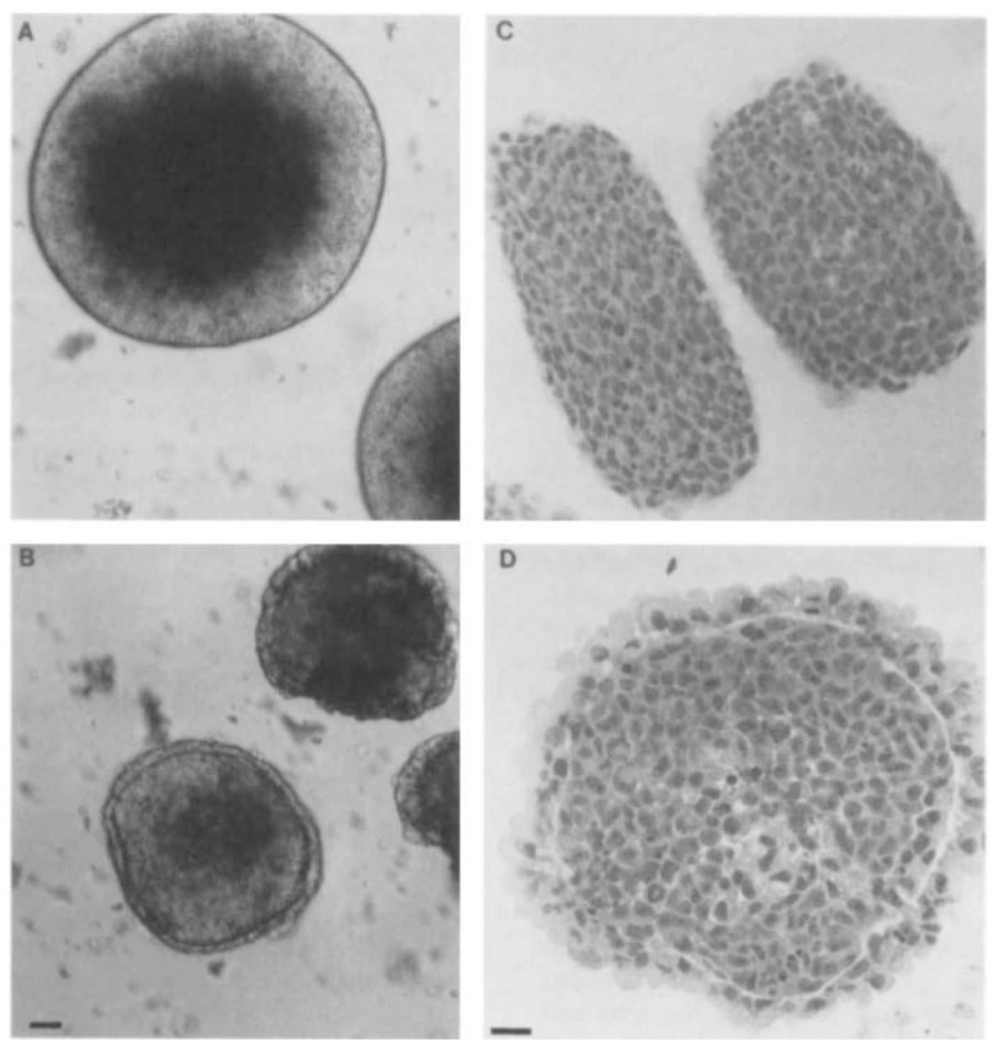

Fig 1.

Morphology of undifferentiated and RA-induced embryoid bodies. A: Bright-field photograph of undifferentiated F9 embryoid bodies. B: Bright-field photograph of RAinduced embryoid bodies. In $\mathbf{C}$ and $\mathbf{D}$ embryoid bodies were embedded in plastic, sectioned, and stained with hematoxylin and eosin. C: Undifferentiated embryoid bodies D: RAinduced embryoid body. Size bar for A and B shown in 9. Size bar for C and D shown in D. Bar $=40 \mu \mathrm{m}$. 

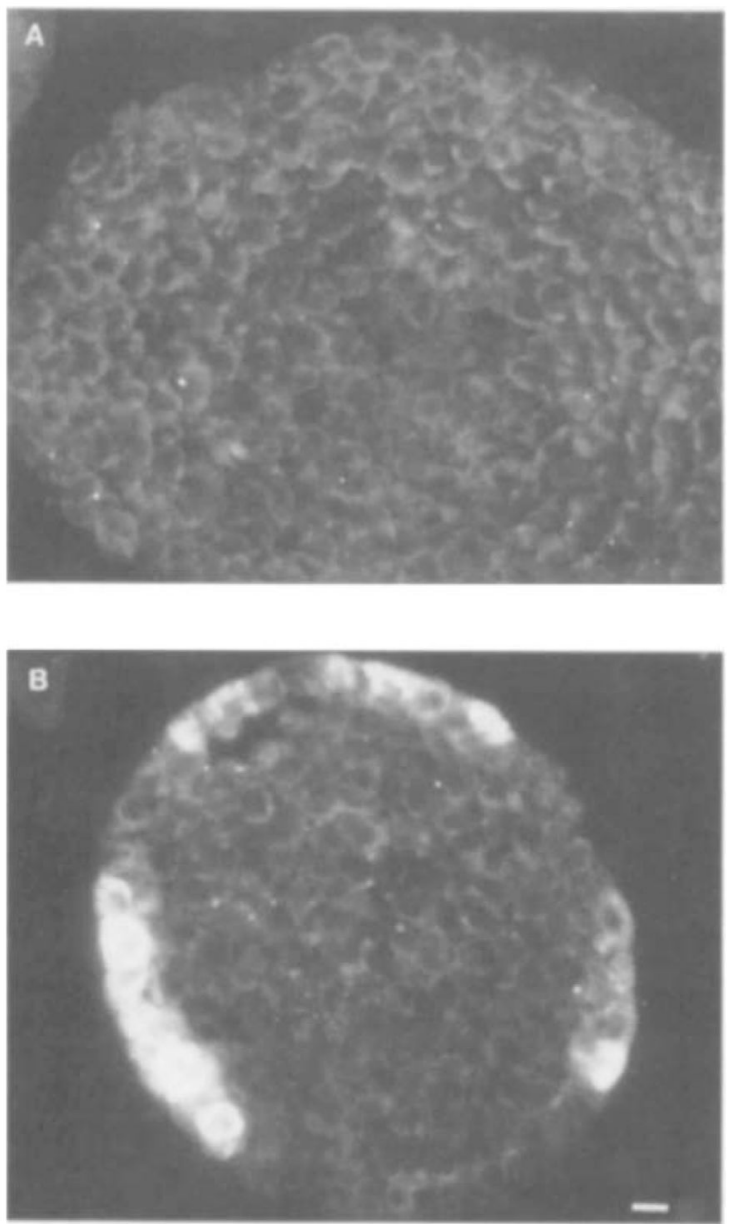

Fig. 2.

Immunocytochemical detection of AFP expression. Embryoid bodies were fixed in acidified ethanol, embedded in paraffin, and stained with a polyclonal antibody specific for AFP. A: Undifferentiated F9 embryoid body. B: RA-induced embryoid body. Bar $=20 \mu \mathrm{m}$. 

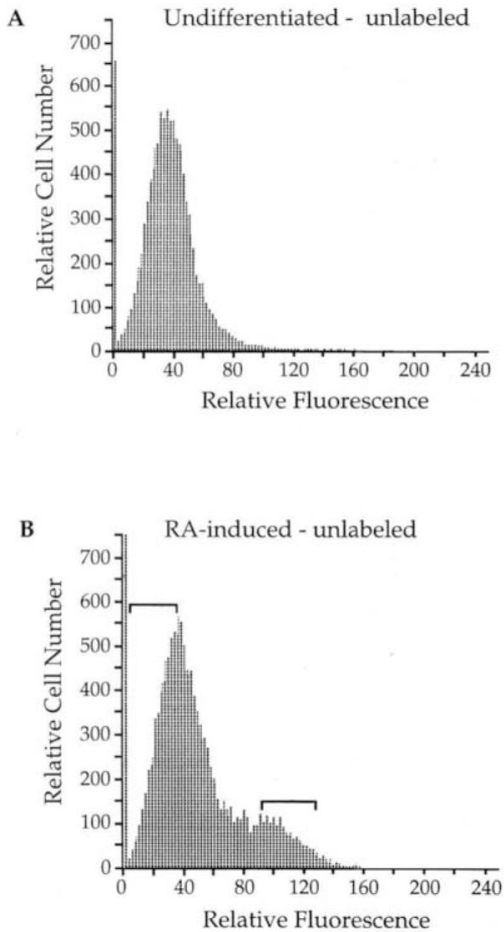

C

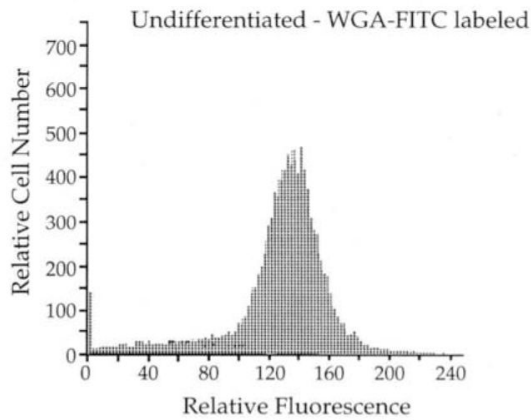

D

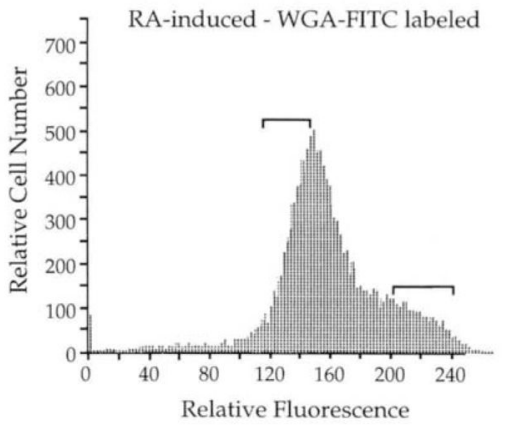

Fig. 3.

Fluorescence profiles of unlabeled and WGA-FITC-labeled embryoid bodies. Cellular fluorescence measurements are presented on a single-parameter logarithmic display. A: Autofluorescence profile of cells from unlabeled, undifferentiated F9 embryoid bodies. B: Autofluorescence profile of cells from unlabeled, RA-induced embryoid bodies. C: Fluorescence profile of cells from WGA-FITC-labeled, undifferentiated embryoid bodies. D: Fluorescence profile of two cell populations from WGA-FITC-labeled, RA-induced embryoid bodies. These profiles were taken from a single collection; however, the same profiles were reproducible over all collection periods. The small signal in the left corner of histograms represents debris resulting from trypsinization of the samples. Brackets indicate sorting windows for the collection of cells. 

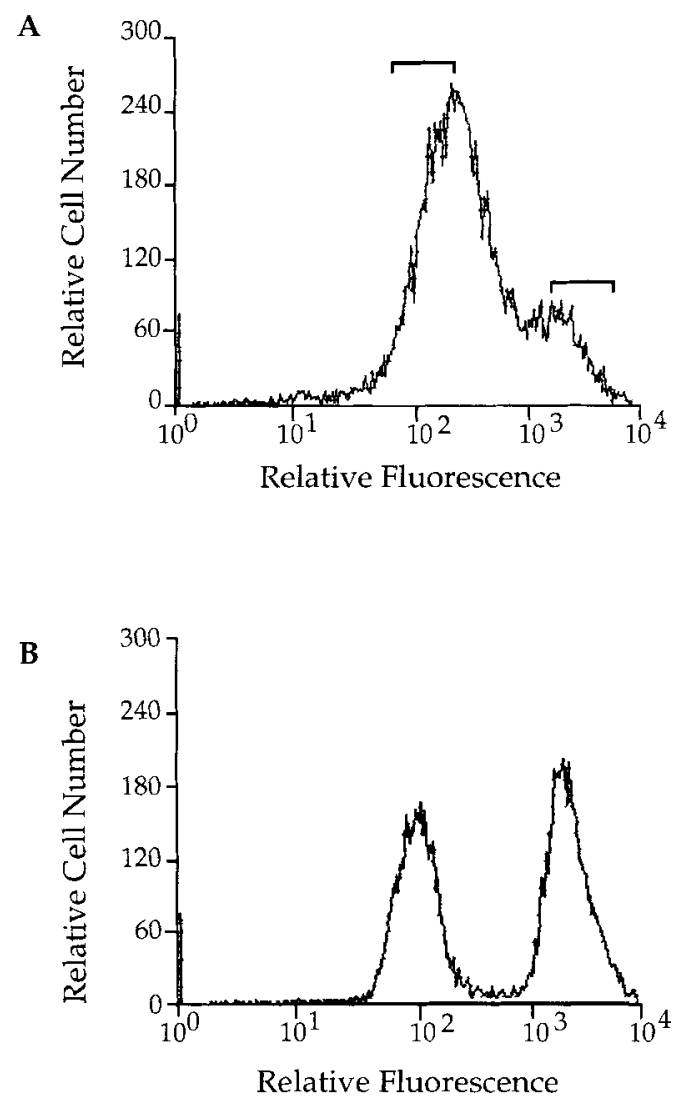

Fig. 4.

Confirmation of sorting parameters. The two cell populations collected from WGA-FITClabeled, RA-induced embryoid bodies were re-analyzed to assess the purity provided by sorting parameters. A: Fluorescence profile of cell populations of WGA-FITC-labeled, RAinduced embryoid bodies before separation and collection. B: Fluorescence profile of the same populations after collected cells were recombined in equal numbers. 


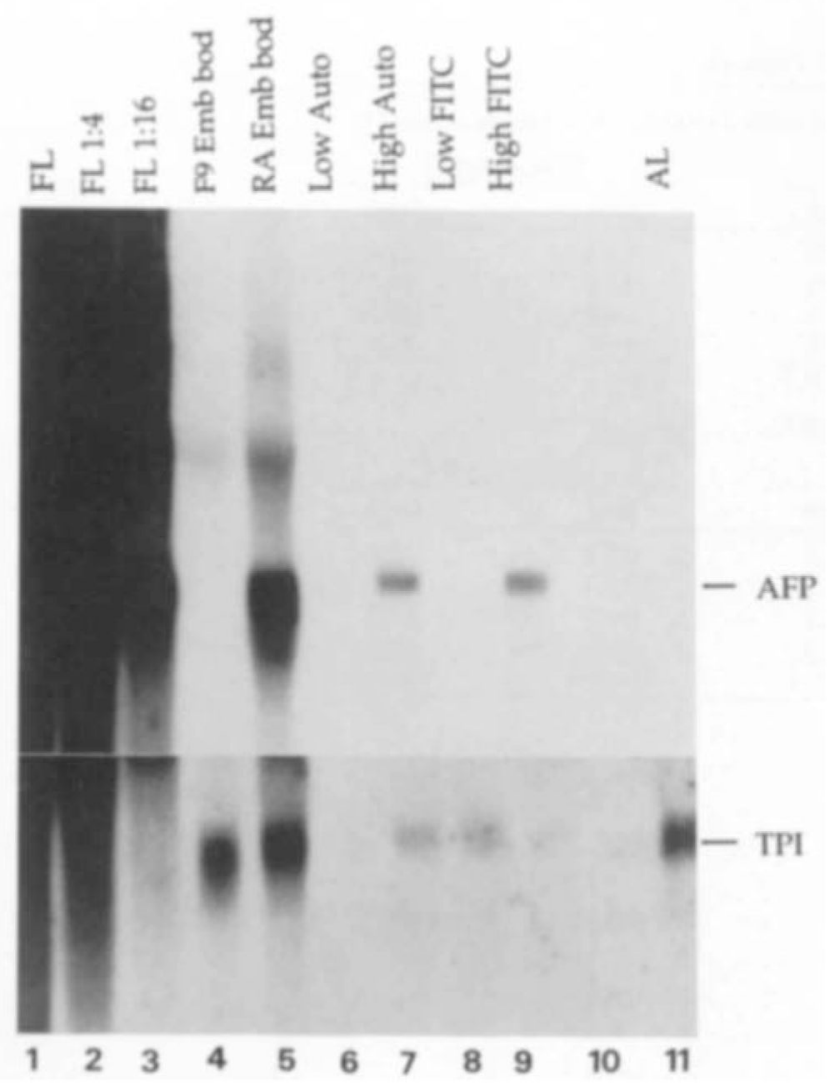

Fig. 5.

RNA analysis of AFP and TPI expression. Total RNA was prepared from fetal liver, adult liver, intact embryoid bodies, and sorted cell populations. RNAs were fractionated by agarose gel electrophoresis, blotted onto nitrocellulose, and hybridized to ${ }^{32} \mathrm{P}$-labeled AFP cDNA (upper panel) or ${ }^{32} \mathrm{P}$-labeled TPI cDNA (lower panel). Lane 1: $15 \mu \mathrm{g}$ of fetal liver RNA; lane 2: 1:4 dilution of fetal liver RNA; lane 3: 1:16 dilution of fetal liver RNA; lane 4: RNA from intact undifferentiated F9 embryoid bodies; lane 5: RNA from intact RAinduced embryoid bodies; lane 6: RNA from the low autofluorescent sorted population of unlabeled, RA- induced embryoid bodies; lane 7: RNA from the high autofluorescent sorted population of unlabeled RA-induced embryoid bodies; lane 8: RNA from the low fluorescent sorted population of WGA-FITC-labeled, RA-induced embryoid bodies; lane 9: RNA from the high fluorescent sorted population of WGA-FITC-labeled, RA-induced embryoid bodies; lane 10: empty lane; lane 11: adult liver RNA. 


\section{Table 1}

Analysis of FACS Profiles

\begin{tabular}{|c|c|c|c|c|}
\hline \multicolumn{5}{|c|}{ A. Peak positions in unstained vs. FITC-labeled cells (relative fluorescence units) } \\
\hline \multirow[b]{2}{*}{ Experiment no. } & \multicolumn{2}{|c|}{ Unlabeled } & \multicolumn{2}{|c|}{ FITC-labeled } \\
\hline & Low & High & Low & High \\
\hline 1 & 59 & 108 & 150 & 211 \\
\hline 2 & 42 & 106 & 154 & 214 \\
\hline 3 & 39 & 111 & 148 & 209 \\
\hline 4 & 49 & 95 & 155 & 216 \\
\hline $\mathrm{x}^{-} \pm$S.D. $=$ & $45 \pm 9$ & $105 \pm 7$ & $152 \pm 3$ & $213 \pm 3$ \\
\hline
\end{tabular}

B. Distribution of cells in peaks (\% of total cells)

\begin{tabular}{|c|c|c|c|c|}
\hline \multirow[b]{2}{*}{ Experiment no. } & \multicolumn{2}{|c|}{ Unlabeled } & \multicolumn{2}{|c|}{ FITC-labeled } \\
\hline & Low & High & Low & High \\
\hline 1 & 64.3 & 34.3 & 61.7 & 37.0 \\
\hline 2 & 70.9 & 26.5 & 75.4 & 24.2 \\
\hline 3 & 64.7 & 27.7 & 66.2 & 32.5 \\
\hline 4 & 63.0 & 32.0 & 61.7 & 37.0 \\
\hline $\mathrm{x}^{-} \pm$S.D. $=$ & $66 \pm 4$ & $30 \pm 4$ & $66 \pm 4$ & $33 \pm 6$ \\
\hline
\end{tabular}

